\title{
Groundwater and contaminant travel time distributions near permeable reactive barriers
}

\author{
H. Klammler ${ }^{1,2,3}$, K. Hatfield ${ }^{1,2}$ \& I. V. Perminova ${ }^{4}$ \\ ${ }^{I}$ Department of Civil and Coastal Engineering, University of Florida, \\ USA \\ ${ }^{2}$ Inter-Disciplinary Program in Hydrologic Sciences, \\ University of Florida, USA \\ ${ }^{3}$ Department of Hydraulic Engineering and Water Resources \\ Management, Graz University of Technology, Austria \\ ${ }^{4}$ Department of Chemistry, Lomonosov Moscow State University, Russia
}

\begin{abstract}
Permeable Reactive Barriers (PRBs) is a passive in-situ technology, which is based on the interception and remediation of a contaminant plume through installation of reactive material in an aquifer. Groundwater and contaminant travel times for a given location in the aquifer to or from the PRB are important parameters in PRB design and performance monitoring. The approach taken is two-dimensional in the horizontal plane and based on existing flow field solutions for a series of PRB configurations. Transport is considered purely advective with a possible retardation between groundwater and contamination. The aquifer is assumed homogeneous and a dimensionless travel time is introduced for arbitrary magnitudes of ambient groundwater flow, confined aquifer thickness and porosity as well as contaminant retardation. Travel time is expressed in a general form by an integral along curved stream lines in the physical plane and transformed into an integral along a straight stream line in the complex potential plane, where a simple numerical integration method is applied to generate maps of isochrones. Travel times are seen to be rather uniformly distributed within capture/release zones with minor effects of local low flow zones (stagnation points). Funnel-and-gate systems show a stronger lateral growth of capture/release zones at early times than other PRB types. Drain-andgate PRBs possess closed isochrones and are identified as transitional configurations between classic PRBs and pump-and-treat systems.
\end{abstract}

Keywords: aquifer, plume, remediation, capture zone, conformal mapping, particle tracking. 


\section{Introduction}

The presence and transport of contaminant plumes in the groundwater is a more than widely recognized threat to the human health and environment. Permeable reactive barriers (PRBs) represent a passive and in-situ alternative to pump-andtreat systems that require a continuous energy supply and commonly include extraction of contaminated groundwater to the surface for treatment (Cunningham and Reinhard [1]). The working principle of PRBs is based on the installation of a reactive medium in the flow path of a contaminant plume, which is thus forced by the natural groundwater gradient to migrate through the reactive medium in the subsurface. Physical, chemical and/or biological processes between the reactive medium and the contaminants lead to the desired degradation and/or deposition of the contaminants as they cross the reactive cell, thus performing the actual remediation process before the groundwater reenters the natural aquifer (EPA [2]). Recent research on uranium and plutonium suggests PRBs could effectively intercept actinide plumes. While a given reactor cell may or may not possess impermeable side walls, the treated portion of the groundwater flow can be increased by deploying impermeable cutoff walls extending from the reactor sides into the aquifer, which is referred to as the funnel-and-gate (FG; Starr and Cherry [3]) system. Velocity equalization walls (VEWs; Painter [4]) have been proposed to achieve a more uniform flux distribution entering the reactor. Furthermore, draining trenches can be used in the drain-and-gate (DG; BBF [5]) configuration, which act as collectors of contaminated groundwater up-gradient of the reactor and as distributors of clean water on the down-gradient side.

A PRB installed by excavation alters the ambient groundwater flow field. Thus, a fundamental issue for the design and operation of a PRB is the hydraulics of the groundwater flow, since it determines both size and location of the capture zone. Moreover, the flow fields near PRBs determine groundwater and contaminant travel times between a given location in the aquifer and the PRB. Travel times are of interest to estimate (1) how long it takes a water or contaminant particle (e.g., at the tail of a finite plume) in the capture zone of a $\mathrm{PRB}$ to reach the reactor and (2) how far down-gradient from the PRB does the body of remediated water reach at a certain time after PRB implementation. The former may be important to assess the required life time of a PRB for complete plume remediation. The latter expresses when a leading (not intercepted by PRB) portion of a plume has passed a down-gradient receptor (e.g., supply well) at a given distance from the PRB or describes the advance of a contaminant front down-gradient of a non-functional (e.g., depleted) reactor.

Fig. 1 gives an example of each of the PRB types considered here ((a) continuous wall "CW"; (b) - CW with impermeable side walls; (c) - FG; (d) PRB with VEW; (e) - DG). Thin continuous stream lines are shown for flow in design ( $x$-) direction corresponding to analytical solutions [6-8 with other applications in 9,10] from a two-dimensional (horizontal) conformal mapping approach. These references also contain a more detailed review of literature related to the topic. Thick dotted lines in fig. 1 represent constant head 
(a)

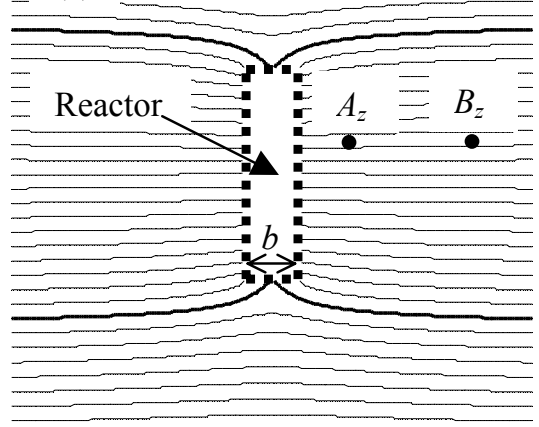

(c)

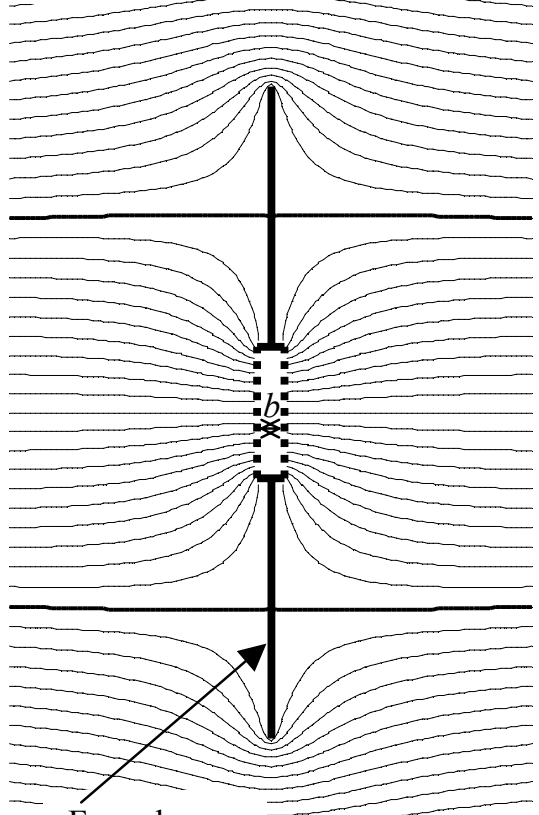

Funnel arm
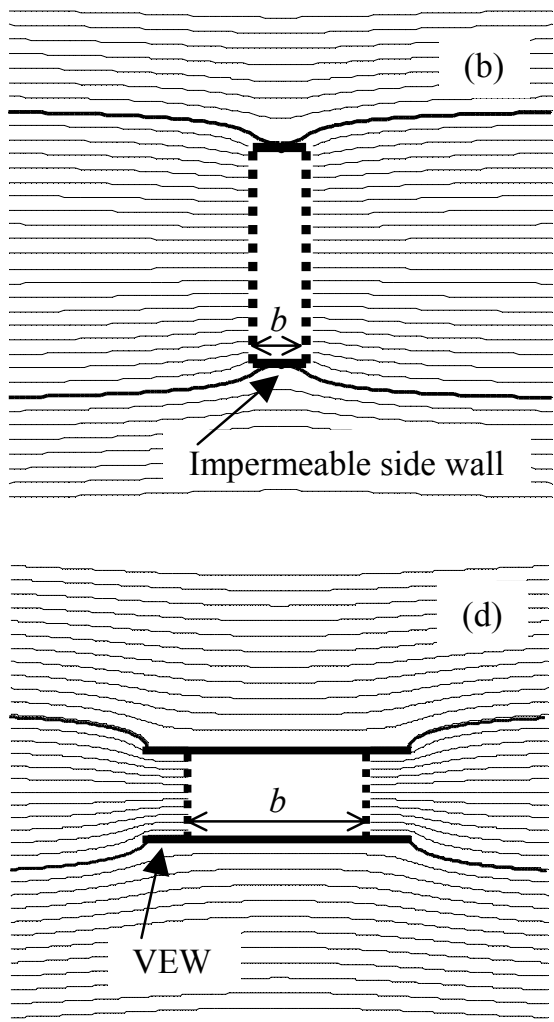

(e)

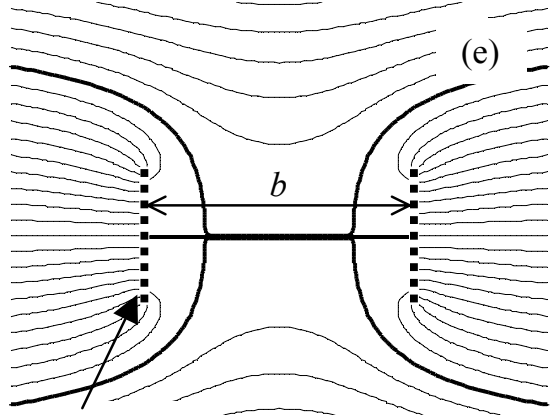

Drain

Figure 1: $\quad$ Examples of PRB types and flow fields.

boundaries (e.g., highly permeable reactor or gravel packs), while thick continuous lines are used for impermeable (e.g., sheet pile) boundaries such as side walls or funnel arms. Thick stream lines delimit the capture zones of each configuration. The goal of the present study is to take advantage of these solutions to compute advective water and contaminant travel times for design 
groundwater flow direction to and from PRBs in dependence of (1) PRB type and dimensions and (2) hydraulic resistance of the reactor. For this purpose, a dimensionless travel time is introduced and results are represented graphically for illustration, validation and discussion.

\section{Theory}

\subsection{Dimensionless particle travel time}

Given a certain flow field and assuming purely advective transport (i.e., neglecting diffusion and dispersion) groundwater or contaminant travel times may be determined by tracking a particle between two points along a stream line [11]. Stream lines are known to be lines of constant stream function as given by [6-8] and local depth integrated Darcy fluxes along each stream line are described by the complex discharge function $W(z)$ [length ${ }^{2} /$ time] [7], where $z=x$ $+i y$ [length] are the complex coordinates of the physical plane. Assuming a homogeneous confined aquifer of thickness $H$ [length], and porosity $n$ [dimensionless] the magnitude of local particle velocity $v=v(z)$ [length/time] may be written as

$$
v=\frac{|W|}{H n R_{d}}
$$

where $|W|$ denotes the absolute magnitude of $W(z)$, and $R_{d}$ [dimensionless] is a retardation factor expressing the ratio between groundwater pore velocity and particle travel velocity. Hence, $R_{d}=1$ for water and $R_{d} \geq 1$ for contaminant travel times due to possible reactive transport (e.g., linear sorption). With this, particle travel time $t_{A B}$ [time] between two arbitrary points $A_{z}$ and $B_{z}$ on a stream line (fig. 1(a)) results as

$$
t_{A B}=\int_{A_{z}}^{B_{z}} \frac{d z \mid}{v}=H n R_{d} \int_{A_{z}}^{B_{z}} \frac{|d z|}{|W|}
$$

where $|\mathrm{d} z|$ [length] is the infinitesimal arc length along a stream line. Eqn (2) may be solved directly in the physical (z-)planes of fig. 1; however, the integration path from $A_{z}$ to $B_{z}$ is generally curvilinear and given by an implicit function. Since the solution for $v$ was obtained by conformal mapping, a complex potential $(\Omega-)$ plane is known for flow in PRB design direction in which flow is uniform from left to right. The mapping from $z$ to $\Omega$ (i.e., $\Omega(z)$ ) is the solution to the flow field problem in terms of potential and stream function, where it is known that $W(z)=\mathrm{d} \Omega(z) / \mathrm{d} z$. With this and introducing $\Omega^{\prime}=\Omega / q_{0}$ [length] as the complex potential for unit depth integrated Darcy flux $q_{0}$ [length ${ }^{2} /$ time] at infinity eqn (2) can be reformulated as

$$
t_{A B}=\frac{H n R_{d}}{q_{0}} \int_{A_{\Omega^{\prime}}}^{B_{\Omega^{\prime}}}\left|\frac{d z}{d \Omega^{\prime}}\right|^{2} d \Omega^{\prime}
$$

where integration from $A_{\Omega}{ }^{\prime}=\Omega^{\prime}\left(A_{z}\right)$ to $B_{\Omega^{\prime}}=\Omega^{\prime}\left(B_{z}\right)$ is now along a straight horizontal stream line (fig. 2). $v_{0}=q_{0} / H n R_{d}$ [length/time] is seen to represent the 
particle velocity at infinity (undisturbed flow domain). According to conformal mapping theory the term $\left|\mathrm{d} z / \mathrm{d} \Omega^{\prime}\right|$ in the integrand of eqn (3) describes the omnidirectional scaling between two corresponding infinitesimal lengths in the $z$ and $\Omega^{\prime}$-planes. Due to the requirement of continuity of flow between corresponding infinitesimal portions of different conformal mapping planes local fluxes are scaled by the reciprocal of this ratio resulting in the fact that $\mathrm{d} t_{A B}$ is proportional to $\left|\mathrm{d} z / \mathrm{d} \Omega^{\prime}\right|^{2}$.

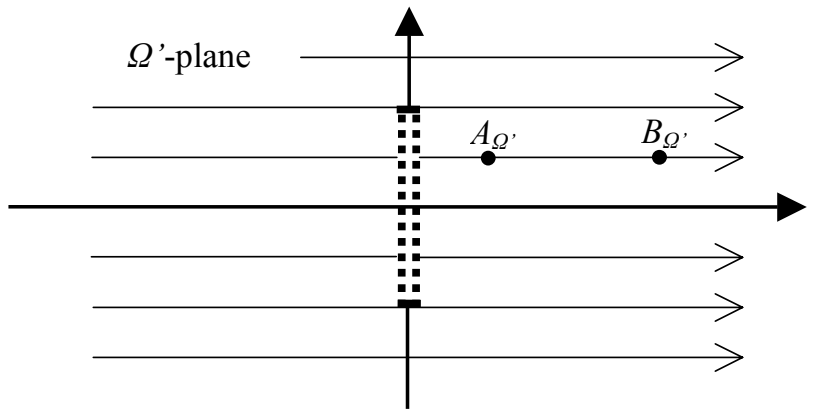

Figure 2: $\quad$ Horizontal stream lines in $\Omega^{\prime}$-plane with image of CW PRB (slot).

For generalization of further analysis the dimensionless particle travel time $t_{A B}^{\prime}$ is introduced as

$$
t^{\prime}{ }_{A B}=\frac{t_{A B} v_{0}}{b}=\frac{1}{b} \int_{A_{\Omega^{\prime}}}^{B_{\Omega^{\prime}}}\left|\frac{d z}{d \Omega^{\prime}}\right|^{2} d \Omega^{\prime}
$$

where $b$ [length] is the PRB (reactor) width as indicated in fig. 1 for each configuration. Intuitively speaking, $t^{\prime}{ }_{A B}$ is a multiple of the particle travel time along a distance $b$ aligned with the undisturbed flow field, i.e., if no PRB had been implemented or very far from it.

\subsection{Continuous wall (CW) PRBs}

For the case of CW PRBs (fig. 1(a)) $\mathrm{d} z / \mathrm{d} \Omega^{\prime}=q_{0}\left(\mathrm{~d} z / \mathrm{d} \tau_{l}\right)\left(\mathrm{d} \tau_{l} / \mathrm{d} \tau_{2}\right)\left(\mathrm{d} \tau_{2} / \mathrm{d} \Omega\right)$ may be written after [6] with

$$
\begin{gathered}
\frac{d z}{d \tau_{1}}=\frac{b k^{2}}{2 \gamma} \sqrt{\frac{\tau_{1}^{2}-1}{k^{2} \tau_{1}^{2}-1}} \\
\frac{d \tau_{1}}{d \tau_{2}}=\frac{\sqrt{k^{2} \tau_{1}^{2}-1}}{k \tau_{1}} \\
\frac{d \tau_{2}}{d \Omega}=\frac{2 \gamma}{q_{0} b k}
\end{gathered}
$$


where $k$ and $\gamma$ are known dimensionless parameters depending on the PRB aspect ratio. Eqn (4), thus, becomes

$$
t^{\prime}{ }_{A B}=\frac{1}{b} \int_{A_{\Omega^{\prime}}}^{B_{\Omega^{\prime}}}\left|1-\frac{1}{\tau_{1}^{2}}\right| d \Omega^{\prime}
$$

where $\tau_{l}\left(\Omega^{\prime}\right)$ is known. However, the resulting expression is not easily integrated and a numerical method as described below is applied using Matlab and its Schwarz-Christoffel mapping toolbox [12].

\subsection{Other PRB types}

For FG, DG and PRBs with VEWs resulting expressions are even more complex and while eqn (4) is generally valid, respective forms of eqn (8) are given for each configuration based on [7,8]. These equations are derived by the same manipulations as for CW PRBs; however, details are omitted for brevity. For FG

$$
t^{\prime}{ }_{A B}=\frac{1}{b} \int_{A_{\Omega^{\prime}}}^{B_{\Omega^{\prime}}}\left|\frac{\tau_{1}^{2}\left(1-\tau_{1}^{2}\right)^{2}}{\left(\tau_{1, H}^{2}-\tau_{1}^{2}\right)\left(e^{2}-\tau_{1}^{2}\right)^{2}}\right| d \Omega^{\prime}
$$

and for PRBs with VEWs

$$
t^{\prime}{ }_{A B}=\frac{1}{b} \int_{A_{\Omega^{\prime}}}^{B_{\Omega^{\prime}}}\left|\frac{\left(\tau_{1, H}^{2}-\tau_{1}^{2}\right)}{\left(e^{2}-\tau_{1}^{2}\right)}\right|^{2} d \Omega^{\prime}
$$

where $\tau_{1, H}$ and $e$ are known mapping parameters depending on PRB geometry and dimensionless reactor hydraulic resistance $R_{h}$. In the absence of funnel arms $\left(\tau_{l, H}=0\right.$ in eqn (9)) or $\mathrm{VEW}\left(\tau_{l, H}=1\right.$ in eqn (10)) both cases reduce to a $\mathrm{CW}$ PRB with impermeable side walls (fig. 1(b)). For DG

$$
t^{\prime}{ }_{A B}=\frac{1}{b} \int_{A_{\Omega^{\prime}}}^{B_{\Omega^{\prime}}} \mid\left(\left.\frac{\left(e_{d}^{2}-\tau_{1}^{2}\right)}{\left(e^{2}-\tau_{1}^{2}\right)}\right|^{2} d \Omega^{\prime}\right.
$$

where $e_{d}$ is also a known parameter depending on drain length and separation. Inspection of eqns (8) through (11) confirms that the integrands become zero at flow singularities (large flow velocities) and infinite at stagnation points (low flow velocities).

\subsection{Numerical integration}

Since the goal is to determine travel times to and from PRBs and since the flow domains considered (and, hence, resulting travel times) are symmetric with respect to both $x$ and $y$-axis (fig. 1), $A_{\Omega}$, in fig. 2 is limited to locations on the positive ordinate with $B_{\Omega}$, anywhere to the right on a respective stream line. By discretizing the first quadrant of the $\Omega$ '-plane into a dense grid of points, which are horizontally separated by a small distance $\Delta s$, and evaluating $\left|\mathrm{d} z / \mathrm{d} \Omega^{\prime}\right|^{2}$ at each point, eqn (4) may be approximated by the sum

$$
t^{\prime}{ }_{A B} \approx \frac{1}{b} \sum_{A_{\Omega^{\prime}}}^{B_{\Omega^{\prime}}}\left|\frac{d z}{d \Omega^{\prime}}\right|^{2} \Delta s .
$$


Knowing $B_{z}\left(B_{\Omega},\right)$ the physical points corresponding to each $t^{\prime}{ }_{A B}$ obtained is found and isochrones (lines of equal travel time) can be interpolated. Using the complex potential plane for numerical integration with constant $\Delta s$ also has the advantage that respective grid points in the physical flow domain are automatically denser populated in high flux areas (e.g., around flow singularities). Note that for $R_{h}>0$ some points in the $\Omega$ '-plane do not possess images in the physical aquifer; respective values of $\left|\mathrm{d} z / \mathrm{d} \Omega^{\prime}\right|^{2}$ are simply set to zero.

\section{Results, discussion and validation}

Fig. 3 displays examples of calculated dimensionless travel times $t_{A B}$ for each PRB configuration considered. Using eqn (4) actual groundwater $\left(R_{d}=1\right)$ and contaminant $\left(R_{d} \geq 1\right)$ travel times for a given location in the aquifer to or from a $\mathrm{PRB}$ are obtained as

$$
t_{A B}=\frac{t_{A B}^{\prime} b H n R_{d}}{q_{0}} .
$$

Capture zones appear as thick continuous lines in the approximate sense of ambient groundwater flow ( $x$-direction). Isochrones inside capture zones are with respect to PRB limits, i.e., thick dotted lines. Isochrones outside capture zones are given for comparison and are with respect to where flow crosses the vertical axis of symmetry ( $y$-axis) outside the PRB. At a sufficient distance from the PRB and as reflected by fig. 3 the flow field becomes undisturbed (uniform) and respective isochrones approach vertical lines of separation $b$ (fig. 1).

Fig. 3(a) represents a simple CW PRB of unit aspect ratio (width/length). While flow singularities at the corners of the PRB do not have a strong effect on travel times, the stagnation point $C$ where flow velocity is zero attracts all isochrones. In other words, contamination at the very margin of the (major) capture zone is expected to reach the reactor much later than contamination approaching the reactor center. However, as demonstrated by fig. 3(a), this "slow fringe" of the capture zone is rather narrow and travel times over the main center part of the capture and release zones are rather uniform. This latter observation is even more pronounced for CW PRBs with impermeable side walls (fig. 3(b)).

Figs. 3(c) and (d) depict a FG configuration of reactor aspect ratio 1:5 and a funnel length equal to reactor length. Fig. 3(c) corresponds to zero hydraulic losses $\left(R_{h}=0\right)$ in the reactor and illustrates how the capture and release zones grow both in $x$ and $y$-direction at small $t_{A B}^{\prime}$. This is due to the magnified capture/release zone by the funnel arms and respective stagnations points on them. Interesting to notice is that the other stagnation points at the intersections between funnel arms and reactor side walls do not show a significant impact on travel times and, hence, the temporal evolution of capture/release zones. Fig. 3(d) represents a case of significant hydraulic losses $\left(R_{h}>>0\right)$ in the reactor, such that capture flow is clearly reduced and respective travel times increased (isochrones closer to reactor). 
Fig. 3(e) corresponds to a PRB with VEW of reactor aspect ratio 1:2 (zero hydraulic losses) and VEW length half of reactor length. Clearly apparent is the effect of VEW in terms of achieving a more uniform flow field and travel time distribution inside (and outside) the capture/release zone. Note that this is the case despite the presence of stagnation points near and flow singularities at the VEW tips.

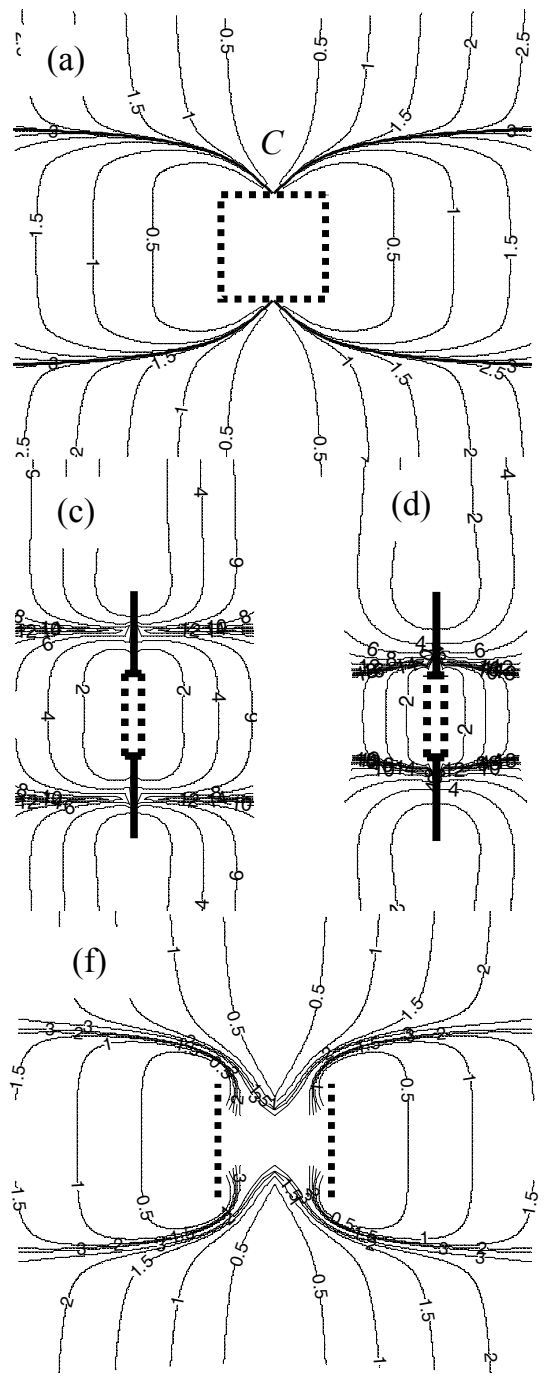

Figure 3: $\quad$ Examples of isochrones of $t^{\prime}{ }_{A B}$.

Figs. 3(f) and (g) represent DG PRBs with drain separation over drain length ratio 1:1. Fig. 3(f) depicts the case of zero hydraulic losses across the reactor and illustrates how isochrones form closed curves around collector and distributor 
drains, similar to what may be expected for injection/extraction wells. In fact, the DG configuration may be regarded as a transition or link between classical PRBs (e.g., CW) and pump-and-treat(-and-reinjection) systems. It is passive by using the siphon principle rather than active pumping; however, the reactor and actual treatment of captured groundwater is completely independent of the PRB structure in the aquifer (e.g., at the surface). Furthermore, "simple" DG PRBs (no distributor drain) may be regarded as some limiting case of a "full" DG PRB where the drain separation is very large. In fig. $3(\mathrm{~g})$ significant hydraulic losses across the reactor are imposed to illustrate the undesirable situation of flow divergence [7] around the reactor. This is reflected by some flow entering the collector drain and rather reentering the aquifer right away than reaching the distributor drain by crossing the reactor. The little spikes on the isochrones outside the capture/release zone are induced by stagnation points on the drains, which separate the portions of incoming and outgoing flow.

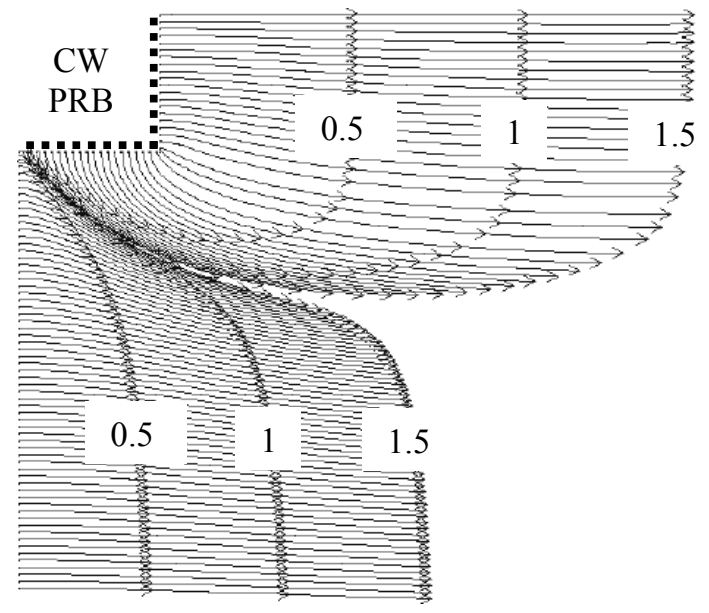

Figure 4: $\quad$ Particle paths and isochrones from numerical simulation.

Results as depicted in fig. 3 were validated against high resolution numerical flow field modeling with particle tracking (MODFLOW, MPATH) and found to be in excellent agreement. Fig. 4 gives an example of particle paths (continuous lines) and respective isochrones of $t^{\prime}{ }_{A B}$ (lines formed by arrow heads) for the case of a CW PRB as shown in fig. 3(a). Fig. 4 is compared to the lower right quadrant of fig. 3(a).

\section{Summary and conclusions}

Groundwater and contaminant travel times for a given location in the aquifer to or from the PRB are important parameters in PRB design and performance monitoring, since they determine (1) when a finite plume has completely passed a PRB, (2) how far the body of remediated water extends down-stream of the 
PRB at a given time after implementation or (3) in the case of PRB mal-function, how the contaminant plume down-stream of the PRB develops with time. While (1) relates to the temporal evolution of the capture zone up-gradient of the PRB, (2) and (3) are concerned with the temporal evolution of the "release" zone down-gradient of the PRB. Moreover, the question arises whether stagnation points (i.e., points of zero local flow velocity) inside or at the margin of capture zones can create "ineffective" capture zones in the sense that respective portions of a contaminant plume will only reach the reactor after an excessively long period of time. This would be the goal for retention of a contaminant plume, but not for remediation.

The approach taken is two-dimensional in the horizontal plane and based on existing flow field solutions [6-8] for continuous wall (CW), funnel-and-gate (FG), drain-and-gate (DG) as well as PRBs with velocity equalization walls (VEW). Transport of groundwater and contaminant particles is considered purely advective (i.e., neglecting effects of diffusion and dispersion), where contaminant transport may be slowed down with respect to groundwater flow by a constant retardation factor. A dimensionless travel time $t_{A B}$ between arbitrary points $A$ and $B$ on a stream line is introduced, which is valid for arbitrary values of ambient groundwater flow magnitude (i.e., background gradient and conductivity), aquifer thickness and porosity as well as contaminant retardation factor. $t_{A B}$ is expressed in a general form by an integral along curved stream lines in the physical plane and transformed into an integral along a straight stream line in the complex potential plane. A simple numerical integration method is applied to compute $t_{A B}^{\prime}$ at a grid of points in the aquifer and to generate maps of isochrones (lines of equal travel times).

For the PRB configurations considered and groundwater flow in PRB design direction resulting travel times are symmetric about both $x$ and $y$-axis and show excellent agreement with validation by numerical flow field modeling in combination with a particle tracking algorithm. For all cases studied the travel times inside the capture/release zone are rather uniformly distributed with only minor effects of slow flow zones at the fringes. Flow singularities (i.e., large local fluxes) do not appear to play a significant role in spatial travel time distributions at the scale investigated. For the FG configuration and early travel times, capture and release zones show a more pronounced lateral growth than other PRB types. An increase in hydraulic resistance of the reactor decreases capture flow and increases travel times near the reactor. VEWs are seen to lead to a more uniform distribution of travel times, while the temporary evolution of capture/release zones of a DG PRB is of the same type as for an extraction/injection well pair, i.e., isochrones form closed curves around drains as long as no flow divergence occurs. This is related to the observation that the DG configuration may be regarded as a transition between classical (e.g., CW) PRBs and pump-and-treat systems.

Results obtained are valid for PRB structures stretching over the entire thickness of a confined aquifer of homogeneous hydraulic conductivity, porosity and thickness. Although field conditions will generally differ from these assumptions the present analysis grants some valuable insight into the properties 
of groundwater and contaminant travel times to and from PRBs. Using eqn (2) the analysis may be extended to groundwater flow directions other than considered here.

\section{Acknowledgements}

This research was partially funded by the Florida Water Resources Research Center under a grant from the U.S. Department of Interior (06HQGR0079) and NATO CELG grant 983197.

\section{References}

[1] Cunningham, J.A. \& Reinhard, M., Injection-Extraction Treatment Well Pairs: An Alternative to Permeable Reactive Barriers. Ground Water, 40(6), pp. 599-607, 2002.

[2] EPA (Environmental Protection Agency), Capstone Report on the Application, Monitoring, and Performance of Permeable Reactive Barriers for Groundwater Remediation: Volume 1, Performance and Evaluation at Two Sites and Volume 2, Long-Term Monitoring of PRBs: Soil and Groundwater Sampling. EPA/600/R-03/045a and b, 2003.

[3] Starr, R.C. \& Cherry, J.A., In-Situ Remediation of Contaminated Groundwater: The Funnel-and-Gate System. Ground Water, 32(3), pp. $465-$ 476, 1994.

[4] Painter, B.D.M., Reactive Barriers: Hydraulic Performance and Design Enhancements. Ground Water, 42(4), pp. 609-617, 2004.

[5] BBF (Bundesministerium für Bildung und Forschung), Anwendung von durchströmten Reinigungswänden zur Sanierung von Altlasten. RUBINHandbuch, BMBF-Vorhaben-Nummer 0271241, 2006.

[6] Klammler, H. \& Hatfield, K., Analytical Solutions for Flow Fields near Continuous Wall Reactive Barriers. Journal of Contaminant Hydrology, 98, pp. 1-14, 2008.

[7] Klammler, H. \& Hatfield, K., Analytical Solutions for the Flow Fields near Funnel-and-Gate Reactive Barriers with Hydraulic Losses. Water Resources Research, 45, W02423, 2009.

[8] Klammler, H., Hatfield, K. \& Kacimov, A., Analytical Solutions for Flow Fields near Drain-and-Gate Reactive Barriers. Submitted to Advances in Water Resources, 2009.

[9] Klammler, H., \& Hatfield K., The Problem of Flow-by-Pass at Permeable Reactive Barriers. In Geo-Environment and Landscape Evolution III, WIT Trans. Built Environ., vol. 100, edited by U. Mander, C. A. Brebbia, and J. F. Martin-Duque, pp. 15-24, 2008.

[10] Kacimov, A., Klammler, H., Constructal Design of Permeable Reactive Barriers: A Groundwater Hydraulics Criterion. Submitted to Journal of Engineering Mathematics, 2009. 
[11] Kacimov, A., Optimization and Analysis of Advective Travel Times beneath Hydraulic Structures. Journal of Hydraulic Engineering, 134(9), pp. 1311-1317, 2008.

[12] Driscoll, T.A., A MATLAB toolbox for Schwarz-Christoffel Mapping. ACM Transactions on Mathematical Software, 22, pp. 168-186, 1996. (toolbox available under: www.math.udel.edu/ driscoll/SC/) 